\title{
Hospitais seguros em desastres: demandas e tecnologias voltadas à redução de riscos
}

\author{
Hospitals safe from disasters: risk reduction demands and \\ technologies
}

Roberto Braz da Silva Cardoso', Alexandre Barbosa de Oliveira1

DOI: 10.1590/0103-11042020E206

RESUMO Ensaio que objetivou analisar a política de 'Hospitais Seguros Frente a Desastres' desenvolvida pela Organização Pan-Americana da Saúde. O estudo discute a importância da interdisciplinaridade e interprofissionalidade na gestão de risco de desastres e traz o Indice de Seguridad Hospitalaria (ISH) como uma tecnologia que, aplicada aos estabelecimentos de saúde, permite o monitoramento contínuo de seu nível de segurança. O ensaio está estruturado em três partes: a primeira aborda o conceito hospitais seguros e as tipologias de vulnerabilidades de estabelecimentos de saúde a desastres; a segunda trata da política de 'Hospitais Seguros Frente a Desastres' no cenário brasileiro; e a terceira apresenta o ISH como tecnologia para a gestão de riscos de desastres. Adiante, destaca a importância capital do setor saúde no contexto social, recomendando que estratégias e ações sejam desenvolvidas para seu pleno e contínuo funcionamento, particularmente no momento em que a população mais precisa buscar o socorro nas unidades de saúde. Conclui-se que o tema 'Hospitais Seguros frente a Desastres’ precisa estar fundamentado na mais ampla discussão interdisciplinar, em práticas interprofissionais colaborativas e na efetiva participação da sociedade, com vistas à consolidação da cultura de segurança em estabelecimentos de saúde no Brasil.

PALAVRAS-CHAVE Hospitais. Segurança. Risco. Emergências. Desastres.

\begin{abstract}
This study aimed to analyze the policy of 'Hospitals Safe from Disasters'developed by the Pan American Health Organization. The study discusses the importance of interdisciplinarity and interprofessionality in disaster risk management and brings the Hospital Safety Index (HSI) as a technology that, once applied to health establishments, allows the continuous monitoring of its level of safety. The article is structured in three parts: the first addresses the concept of safe hospitals and the typologies of vulnerabilities of health facilities to disasters; the second deals with the 'Hospitals Safe from Disasters' policy in the Brazilian scenario; and the third presents the HSI as a technology for disaster risk management. The article emphasizes the importance of the health sector in the social context, recommending that strategies and actions be developed for its full and continuous functioning, particularly when the population needs to seek assistance in health facilities. It is concluded that the theme 'Hospitals Safe from Disasters' needs to be grounded in the broadest interdisciplinary discussion, collaborative interprofessional practices, and the effective participation of society, with a view to consolidating the safety culture in health facilities in Brazil.
\end{abstract}

KEYWORDS Hospitals. Safety. Risk. Emergencies. Disasters.

1 Universidade Federal do

Rio de Janeiro (UFRJ) - Rio de Janeiro (RJ), Brasil. alexbaroli@gmail.com 


\section{Introdução}

Os fenômenos naturais e a ação do homem sobre a natureza produzem alterações de maior ou menor magnitude em todas as regiões do planeta, inclusive destruindo ecossistemas. Tais eventos adversos deixam em alerta as autoridades locais para o acionamento de planos de contingência para população civil e desencadeamento de atendimento emergencial a ela.

O conjunto de estabelecimentos de saúde que compõem o sistema de saúde local, regional e nacional é convocado a participar nas diversas linhas de suas áreas de atuação. No entanto, essas instituições e seus diversos recursos também podem estar vulneráveis e sofrer com as consequências dos desastres, que podem danificar ou destruir suas instalações, limitando ou impedindo o seu funcionamento, justamente quando deveriam estar em condições plenas de responderem a uma situação de emergência, em que a demanda dos cidadãos por serviços de saúde em muito pode exceder sua capacidade de atendimento.

No Brasil, o tema 'hospital seguro' (em contexto de desastres) ainda é pouco difundido; em consequência, pode-se presumir que muitas construções prediais hospitalares, públicas e privadas estejam vulneráveis a tais eventos e que, em virtude da ocorrência deles, tenham reduzidos ou interrompidos a sua capacidade e o seu potencial para socorrer as múltiplas vítimas, na medida em que poderão ser duramente atingidas pelos efeitos dosdesastres causados sejam por fenômenos naturais ou pela ação humana.

As inundações e os deslizamentos, que têm assolado diversas partes do território brasileiro, destacam-se como desastres de origem natural de grande impacto, gerando danos irreparáveis à população, especialmente quando as unidades de saúde são também atingidas. $\mathrm{O}$ mesmo se pode afirmar em relação às epidemias, implicando aumento extra de atendimento nos serviços de saúde, impossível de ser sustentado sem o adequado preparo destes, como ocorreu na epidemia de dengue no Rio de Janeiro em 2008, quando os órgãos públicos, sem condições de suprir a repentina elevação da demanda, necessitaram recorrer a 'ações de guerra', por meio da instalação de barracas de campanha'.

Diante dessas circunstâncias, é importante destacar os aspectos interdisciplinarese interprofissionaispara a consolidação da cultura de segurançaem cenário hospitalar, inclusive nos contextos de desastres. Na medida em que os saberes se articulam e interagem entre si, em torno de um tema no qual a participação de cada profissional envolvido se soma e se potencializa, reforça-se o conceito de hospital seguro, uma vez que este resulta não da ação isolada de um saber hegemônico, mas da conjunção de vários saberes, englobando não somente aqueles diretamente relacionados com a assistência hospitalar (enfermagem, farmácia e medicina por exemplo), mas também os relacionados com a geração e com a manutenção de importantes condições para que a assistência ocorra (administração, estatística, geografia, engenharia e arquitetura por exemplo).

A ocorrência de desastres vem aumentando nos últimos anos, exigindo maior adesão na participação de profissionais de diversas áreas, de diferentes estados, regiões e países para seu enfrentamento, de maneira a criar um trabalho efetivamente conjunto, com integração entre diversas profissões e atores envolvidos, que precisam dar conta da multiplicidade de demandas, auxiliando-se mutuamente. Percebese, por conseguinte, a necessidade de inserção de novas tecnologias para atuar de maneira interdisciplinar, visando a um objetivo comum que são os atendimentos requeridos por cada evento em especial.

Eis, portanto, um grande desafio a ser vencido diante das multidimensionalidades humanasem contextos de desastres: ir além dos modelos já ultrapassados, hierarquizados e não articulados de saberes, para que se alcance a proposta de fluidez da comunicação e ação entre saberes que se complementam no 
cenário desafiador dos desastres e emergências, tanto em seu contexto amplo quanto ao aplicado à realidade da saúde.

Desse modo, o Marco de Sendai, que foi assinado em março de 2015 no Japão durante a III Conferência Mundial para a Redução de Risco de Desastre 2015-2030, é um elemento que justifica este estudo. Dentro de suas prioridades de ação, o Marco de Sendai, do qual o Brasil é país signatário, preconiza a compreensão do risco de desastres e enfatiza a importância de campanhas globais e regionais, como, por exemplo, a iniciativa 'Um milhão de escolas e hospitais seguros'2

É a partir de tais ideias e apontamentos que este estudo objetivou analisar a política 'Hospitais Seguros Frente a Desastres' desenvolvida pela Organização Pan-Americana da Saúde (Opas). Nesse sentido, o presente ensaio está estruturado em três partes: a primeira aborda o conceito hospitais seguros e as tipologias de vulnerabilidades de estabelecimentos de saúde a desastres; a segunda trata dessa política no cenário brasileiro; e a terceira apresenta o Indice de Seguridad Hospitalaria (ISH) como tecnologia aplicável para a gestão de riscos de desastres.

\section{Discussão}

\section{Hospitais seguros e vulnerabilidade a desastres}

A Opas define 'hospital seguro' como um estabelecimento de saúde cujos serviços permanecem acessíveis e em funcionamento, em sua máxima capacidade instalada e na mesma infraestrutura, imediatamente após fenômenos destrutivos de origem natural ou tecnológica, ainda que esse mesmo hospital tenha sido atingido; e também na fase de recuperação dessas emergências, uma vez que a continuidade do funcionamento de todo o conjunto nosocomial é vital para o atendimento seguro às pessoas atingidas. Assim, o termo 'hospital', na acepção de 'hospitais seguros frente a desastres', é utilizado com o propósito de facilitar a identificação de um serviço de saúde concreto, em amplo sentido, referindo-se a todos os estabelecimentos de saúde, independentemente do porte ou complexidade ${ }^{3}$.

Em geral, o funcionamento ininterrupto dos serviços de saúde costuma marcar a diferença entre a vida e a morte; portanto, é prioritário garantir que todos esses serviços contem com uma edificação resistente aos choques dos fenômenos de origem natural ou tecnológica, que seus equipamentos não sofram danos, que suas linhas vitais (água, eletricidade, gases medicinais etc.) sigam funcionando, e que seu pessoal seja capaz de continuar oferecendo assistência de saúde nos momentos em que mais necessita. Com efeito, ser capaz de manter-se em pleno funcionamento nas circunstâncias adversas típicas dos desastres é o que define um hospital seguro ${ }^{3}$.

É consenso que os hospitais são organizações essenciais destinadas a proporcionar atenção à saúde com garantia de eficácia, eficiência, oportunidade e qualidade. A obrigatoriedade de dedicar atenção, oferecer e realizar assistência de saúde universal, integral e equânime aos usuários que dela necessitem tem conotações técnicas, administrativas, éticas e penais. Para que um hospital seja seguro e siga funcionando, inclusive durante a ocorrência de um desastre, certas características da edificação e sua funcionalidade devem ser avaliadas quanto à sua vulnerabilidade.

Os estabelecimentos de saúde no conjunto, especialmente os hospitais, requerem uma gama de recursos humanos, materiais, econômicos e tecnológicos. Tais recursos integrados se relacionam com as suas estruturas e processos, de tal forma que o que afeta um elemento tem repercussão no conjunto e no produto, pois todos concorrem para o alcance dos melhores resultados. Nesse sentido, a relação entre as áreas que compõem os estabelecimentosde saúde pode garantir não só um adequado funcionamento em condições de normalidade como também em casos de emergências e desastres ${ }^{4}$. 
Tomando como base essas considerações, muitos países do continente americano têm contemplado a questão da segurança hospitalar em desastres nas suas políticas públicas de saúde, instados pelo histórico da frequente ocorrência de ameaças naturais, tais como abalos sísmicos, furacões e tempestades que geram grandes inundações, nos quais é grande o número de pessoas desabrigadas, acidentadas e doentes, as quais procuram socorro nos estabelecimentos de saúde, que devem estar aptos a atender a uma demanda aumentada. Para tanto, é necessário primeiramente que os estabelecimentos de saúde resistam àqueles eventos, e que mantenham preservados todos os componentes estruturais, não estruturais e funcionais.

O reconhecimento da vulnerabilidade desses componentes é um aspecto estratégico para a identificação de riscos e controle de danos, permitindo que medidas de mitigação possam ser implementadas de acordo com os recursos disponíveis 5 . O quadro 1 a seguir descreve essas diferentes tipologias de vulnerabilidades, que podem ser observadas em estabelecimentos de saúde:

Quadro 1. Condições de vulnerabilidade em estabelecimentos de saúde diante de situações de emergências e desastres

\begin{tabular}{ll}
\hline Condição de Vulnerabilidade & Descrição \\
\hline Vulnerabilidade estrutural & Refere-se às partes do edifício hospitalar que o mantêm de pé, incluindo funda- \\
& ções, colunas, paredes de suporte, vigas e lajes. A falha de um destes elementos \\
& pode causar sérios problemas ao edifício, até mesmo a destruição completa. \\
Vulnerabilidade não estrutural & Refere-se aos componentes do edifício que estão ligados às peças estruturais, \\
& como, por exemplo, tabiques, janelas, tetos, portas, paredes etc.; aos componentes \\
& que desempenham funções essenciais na construção, como calefação, ar condi- \\
& cionado, ligações elétricas etc.; e aos componentes que estão dentro da estrutura, \\
& como os equipamentos médicos, equipamentos mecânicos, móveis etc. Esses \\
& elementos oferecem os serviços básicos de sistemas de água, saneamento, dre- \\
& nagem de águas pluviais, instalações elétricas, sistemas de comunicações, gases \\
& medicinais e sistemas elétricos contra os incêndios, que são essenciais para o \\
& funcionamento de uma unidade de saúde, além dos equipamentos para diagnósti- \\
& co e tratamento das vítimas do desastre. \\
& Refere-se à distribuição e à relação entre espaços arquitetônicos e serviços clíni- \\
& cos e de apoio dentro do hospital, bem como aos processos administrativos de \\
& contratações, compras, rotinas de manutenção etc., e às relações de interdepen- \\
& dência física e funcional entre as diferentes áreas de um hospital. Um adequado \\
& zoneamento e relação entre as áreas que compõem a instalação podem garantir \\
uma operação eficaz, não só em condições normais, mas também em caso de & \\
emergências e desastres.
\end{tabular}

Fonte: Organización Panamericana de la Salud. ¿Su hospital es seguro? Preguntas y respuestas para el personal de salud. Quito: OPS; 20075.

Em publicação sobre hospitais seguros, foi ressaltado que as unidades de saúde das Ilhas Cayman (Caribe) resistiram aos ventos fortes, chuvas torrenciais e furacões e mantiveram-se intactas e em condições de atender às necessidades de uma população de atingidos; os danos registrados envolveram componentes não estruturais. A mesma publicação reitera a necessidade de investimento em reforços da estrutura predial hospitalar, ao citar o exemplo do terremoto de magnitude 6,9 na escala Richter que atingiu a Costa Rica em 1990, quando naquela ocasião alguns prédios hospitalares estavam sendo reforçados. Isso resultou que os edifícios ou parte deles, que haviam sido reforçados, resistissem e não sofressem 
abalos, mas a parte ainda não reforçada ficou estruturalmente danificada. Prossegue citando um exemplo em El Salvador, quando, em 2001, um hospital infantil reforçado em sua estrutura predial resistiu a um forte terremoto, sofrendo apenas danos estéticos ${ }^{5}$.

O aumento contínuo das perdas ocorridas durante e após os desastres e o impacto crescente destes nas economias não são associados simplesmente a um aumento do número de eventos naturais extremos, mas na elevação constante do número da população em áreas sob ameaças, sem correspondente infraestrutura. As comunidades mais vulneráveis são suscetíveis de sofrer danos e perdas de maior magnitude, enfrentando sérios desafios para se recuperar. A inexistência ou a precariedade de planejamento, aliadas à falta de investimento efetivo em saúde pública e à preocupante forma de intervenção no meio ambiente, dão margem ao surgimento e reforço de novas ameaças do tipo socioambiental. Assim, a vulnerabilidade interage com as ameaças, produzindo novas condições de risco, dimensionadas de diferentes formas em cada território ${ }^{6}$.

No bojo da interação existente entre risco, ameaça e vulnerabilidade, o risco expressa a relação existente entre a probabilidade de que uma ameaça de evento adverso ou determinado acidente se concretize, com o grau de vulnerabilidade do sistema receptor aos efeitos dele decorrentes. Convencionalmente, o risco é expresso pela fórmula: Risco $=$ Ameaça $\mathrm{X}$ Vulnerabilidade. De modo geral, esse dispositivo mnemônico não representa necessariamente uma equação matemática a ser utilizada para fins de cálculo, mas apresenta o risco de desastre como uma relação entre ameaças e vulnerabilidades. Além disso, o risco de desastre tende a ser menor pela capacidade individual e local de prevenção e resposta ou por ações de proteção realizadas por entidades maiores, como governo e setores ${ }^{7}$.

Cabe enfatizar que, quando há um aprofundamento sobre o tema, observa-se que os terremotos, furacões e tsunamis não representam as únicas ameaças possíveis ao funcionamento do estabelecimento de saúde ou à segurança da comunidade, contrariando o senso comum equivocadamente estabelecido.

Dados apurados pela Opas revelaram, a partir das informações de seus EstadosMembros, que cerca de 67\% dos hospitais do continente americano estão localizados em zonas de risco para desastres, frisando que, em média, um hospital que não funciona, ou que tem sua infraestrutura funcionando precariamente, deixa cerca de 200 mil pessoas sem atenção em saúde; e a perda dos serviços de urgência durante os desastres diminui drasticamente as chances de salvar vidas. Sendo assim, a manutenção da operacionalidade do hospital deve ser prioridade, uma vez que o atendimento aos cidadãos atingidos não deve ser reduzido ou paralisado ${ }^{8}$.

Nesse contexto, os danos que podem ser provocados nos estabelecimentos de saúde estão relacionados não só com as ameaças presentes, com a localização do estabelecimento, com a topografia do terreno, com sua geologia, com os materiais empregados e com a tecnologia aplicada à construção predial, mas também com seu estado de conservação ao longo do tempo, além de outros fatores possíveis. Vale ressaltar que as unidades de saúde de menor complexidade geralmente são as mais atingidas, uma vez que, comumente, dispõem de menos recursos e infraestrutura para lidar com os efeitos de ocorrências inesperadas ${ }^{6}$.

A ausência de informação precisa e atualizada sobre a quantidade e condições de segurança do recurso físico em saúde e a existência de serviços de saúde dispersos e de difícil acesso têm dificultado sua quantificação no que se refere ao nível de segurança e ao impacto de sua vulnerabilidade. Portanto, conhecer esse nível antes que ocorra o desastre com o objetivo de identificar os elementos que requerem melhorias assim como também priorizar a intervenção nas instalações que, por sua natureza, localização ou importância, devem seguir em funcionamento durante um desastre caracterizam os passos iniciais de gestão na temática 'hospitais seguros'. 


\section{O cenário nacional}

Para o contexto brasileiro, a III Conferência Mundial para a Redução de Risco de Desastreocorrida em Sendai, no Japão, reforçouoportunamente a necessidade de uma maior integração entre os setores de Saúde e Proteção e Defesa Civil, aliando também esforços conjuntos de outros órgãos e instâncias públicas e privadas para o alcance de medidas preventivas e assistenciais consistentes, para lidar com os desastres e suas repercussões.

Campanhas e iniciativas, particularmente no âmbito da saúde pública, devem ser implementadas nas comunidades com o intuito de disseminar as ações de gestão de riscos de desastres. Assim, o treinamento dos agentes comunitários de saúde, por exemplo, pode colaborar para que se obtenha um diagnóstico da quantidade de pessoas e suas características em suas respectivas áreas de risco, contribuindo para um estudo de demanda antecipada. $\mathrm{Na}$ vigência de um evento adverso, ações podem ser adotadas mais pronta e efetivamente.

No que diz respeito à temática envolvendo hospitais seguros em desastres no Brasil, um estudo apontou que, de três hospitais analisados no noroeste do estado do Rio de Janeiro, dois deles foram construídos margeando rios distintos da região ${ }^{9}$. Outra pesquisa observou que as inundações em Santa Catarina (2008) e Pernambuco (2010) provocaram a destruição e a danificação de hospitais e postos de saúde, com prejuízos diretos que demandaram investimentos financeiros elevados para reconstrução e deixaram a população necessitada desassistida ${ }^{10}$.

Com efeito, são ainda pouco expressivos os estudos e as ações nacionais sobre o tema, o que muito provavelmente resulta da cultura brasileira tipicamente reativa e avessa à gestão preventiva, em que os desastres não são tratados como prioridade. Também é fato o senso comum entre os brasileiros de que 'o problema nunca vai ocorrer', sobre a crença instalada de não se atribuir a devida importância aos instrumentos de planejamento, prevenção e avaliação, além das dificuldades de financiamento de estudos acadêmicos de impacto.

Acrescente-se ainda a essas justificativas a equivocada concepção generalizada entre os brasileiros de que, no Brasil, os desastres quase não acontecem, fruto da ideia que se tem de desastres exclusivamente associados a grandes catástrofes como as causadas por terremotos, vulcanismos e tsunamis, desconsiderando os amplos efeitos de fenômenos mais comuns no País, como a seca, a estiagem e as enxurradas, as inundações, os deslizamentos, além dos desastres ligados a causas sociais.

Hoje se sabe que os desastres são multidimensionais, afetando muitas vertentes e resultando de diversos fatores que se conjugam. $\mathrm{O}$ termo 'desastres naturais', por exemplo, não expressa mais a realidade, pois o fenômeno que gera a ocorrência pode até ser da natureza, mas longe de não sofrer a influência humana, por intermédio de suas ações econômicas, políticas e sociais ${ }^{11,12}$.

É notório constatar a grande repercussão que os fenômenos sociais que acabam por caracterizar desastres de destacadas proporções vêm revelando. Assim, eventos de origem social convergem para compor um panorama multicausal de catástrofes humanas contemporâneas. Nesse cenário, impõem-se a violência urbana com seus vários matizes e consequências. Os desastres aí criam raízes, robustecem-se e multiplicam-se, fortalecendo suas sementes pelo incremento da indiferença social a anunciar suas próprias catástrofes ${ }^{13}$.

Um estabelecimento de saúde que está localizado em área classificada como de risco elevado para a violência urbana, ou próximo a ela, poderá ter acesso restrito às suas instalações pela comunidade que dele necessita. $\mathrm{O}$ fato torna-se mais preocupante em situações relacionadas com os desastres, uma vez que não somente o acesso da população atingida estará dificultado ao estabelecimento como certamente a própria unidade assistencial não se encontrará em condições de receber as pessoas atingidas, fruto de grande período sem o devido preparo de seu quadro funcional 
e instalações à mercê da desassistência do serviço público oficial e controle do poder 'paralelo' de supostas organizações criminosas ${ }^{\mathbf{1 4}}$.

Os deslocamentos massivos de pessoas é outro fenômeno social importante nos dias atuais. Geralmente, eles não são precedidos pelo adequado preparo para receber os ' $\mathrm{mi}$ grantes forçados', trazendo como consequência um aumento acentuado da demanda por assistência social, incluindo a saúde. $\mathrm{O}$ panorama tende a piorar quando, ao quadro já preocupante das aglomerações, sobrevêm as epidemias e mais violência.

No Brasil, em apuração feita entre os anos 2000 e 2017, há, pelo menos, 8,8 milhões de pessoas deslocadas forçadamente, em quatro categorias: refugiados, violência, desenvolvimento e desastres. A grande maioria delas - cerca de 6,4 milhões - teve que abandonar os seus lares devido a desastres de origem natural ou provocados pelo homem, como incêndios e rompimentos de barragens. Os projetos de desenvolvimento, por sua vez, deslocaram pelo menos 1,2 milhão de brasileiros nos últimos 18 anos. Já a violência em espaços rurais forçou o deslocamento de cerca de 1,1 milhão de pessoas. No que diz respeito aos refugiados, identificou-se que o Brasil acolhe apenas 10.022 deles ${ }^{15}$.

Assim como no Brasil, os deslocamentos são sentidos em várias regiões do planeta. Mais recentemente, chamam atenção os casos dos refugiados africanos rumo à Europa e dos venezuelanos que atravessam a fronteira noroeste do Brasil, buscando refúgio no estado de Roraima, na esperança de melhores condições de sobrevivência.

A crise humanitária na Venezuela e a consequente migração massiva de cidadãos daquele país para o estado de Roraima fizeram ressurgir casos de sarampo, doença que estava erradicada do Brasil desde 2015. Com as aglomerações de venezuelanos em acampamentos improvisados nas praças e ruas daquele estado, as autoridades sanitárias locais revelaram preocupação com o surgimento de novas epidemias e com a incapacidade do sistema de saúde local para o atendimento à crescente demanda dos deslocados ${ }^{16}$.

A complexidade e a multiplicidade de fatores, condições e consequências relacionadas com o desastre fazem dele multidimensional e necessariamente de abordagem interdisciplinar e interprofissional, envolvendo distintas áreas de conhecimento, ofícios e setores da sociedade. As políticas públicas de saúde devem estar engajadas e basear-se no monitoramentoe planejamento contínuo de ações, para evitá-lo ou mitigar seus efeitos sobre as populações.

Ademais, a intervenção desmedida do homem sobre a natureza, por meio dos desmatamentos, geração de resíduos, poluição de mananciais e ocupação desordenada do solo urbano, tem gerado efeitos sérios no clima do planeta e provocado maior ocorrência e impacto de desastres sobre as comunidades, principalmente naquelas mais vulneráveis ${ }^{12}$.

Em adição à negativa intervenção do homem sobre o ambiente nos países industrializados, historicamente, as políticas públicas não intervêm de maneira a minorar os impactos da degradação. A falta de investimentos em saúde, a burocracia e a cultura imprevidente somam-se e propiciam um cenário no qual os efeitos dos desastres potencializam-se. Nos países em desenvolvimento, como é o caso do Brasil, tais condições se acentuam e criam uma equação funesta e favorável para a perpetuação do cotejo de perdas e dor associado às catástrofes.

Urge, portanto, que nos dediquemos à gestão de risco de desastres, empregando decisões administrativas, de organização e co-nhecimentos operacionais desenvolvidos pelas sociedades e comunidades para implementar políticas, estratégias e fortalecer suas capacidades, a fim de reduzir o impacto de ameaças naturais e de desastres ambientais e tecnológicos consequentes ${ }^{6}$.

No setor saúde, além de um necessário desenvolvimento de ações integradas em todos os níveis de ação, é fundamental a aplicação de tecnologias que colaborem para preparar os estabelecimentos de saúde para continuarem 
prestando plenamente o atendimento à população porventura atingida por desastres. Manter os estabelecimentos de saúde em funcionamento em tais circunstâncias é vital. É para essas unidades de saúde, sejam quais forem, grandes ou pequenas, que as pessoas atingidas correm ou são evacuadas na procura de socorro, devendo as referidas unidades estarem previamente em condições de responder à demanda.

\section{O ISH como tecnologia de gestão de risco de desastres}

Existem diversas ferramentas para realizar estudos de vulnerabilidade, baseados em métodos qualitativos ou quantitativos, mas somente algumas delas são específicas para estabelecimentos de saúde, que, por suas características de funcionamento e de provisão de serviços, acabam por estar especialmente mais vulneráveis, não por serem estruturas mais frágeis, mas por não poderem perder ou ter prejudicada sua efetividade de atendimento. Assim mesmo, durante um tempo, promoveu-se o desenvolvimento de complexos estudos de vulnerabilidade, como se realizou no Equador e em diversos outros lugares, mas dados o seu alto custo e a falta de operacionalização, optou-se por gerar ferramentas que, de uma forma mais simples e menos onerosas, permitam conhecer a capacidade de resposta hospitalar em desastres $\mathbf{1 4 , 1 7}$.

Foi com a perspectiva de desenvolver e reforçar a cultura de hospitais seguros em desastres que a Opas, em 2008, com o apoio de um grupo de especialistas de diferentes países, elaborou o ISH como uma ferramenta de avaliação rápida e confiável, que proporciona uma ideia imediata do nível de segurança de um hospital, como instalação que deve seguir funcionando logo após um evento adverso ${ }^{3}$.

Em 2010, diante dos resultados satisfatórios quanto à utilização do ISH para hospitais, a Opas lançou nova versão para estabelecimentos de saúde de menor porte, de até 20 leitos ou sem internação, mantendo os objetivos da edição original ${ }^{17}$.
O ISH é um instrumento técnico de medição que, por meio de uma lista de verificação e um modelo matemático, leva em consideração a localização geográfica, a estrutura do edifício com seus componentes de formação e a organização do hospital, para obter um valor numérico que aporta uma ideia da probabilidade que tem um estabelecimento de saúde de continuar funcionando depois de um desastre. No entanto, esse índice não substitui uma avaliação exaustiva da vulnerabilidade, mas pode-se utilizá-lotambém como sistema qualitativo de avaliação, que facilita a priorização de ações nas instalações de saúde, uma vez que fornece insights para aspectos passíveis de análise para além do índice em si $\mathbf{1 4 , 1 7}$.

O ISH oferece um parâmetro inicial para que sejam estabelecidas prioridades de investimentos e para que as autoridades possam diagnosticar, de maneira mais ágil, as esferas nas quais seria mais eficiente uma intervenção, visando ampliar a segurança e reconhecer as vulnerabilidades presentes, de forma a planejar e preparar as unidades de saúde para respostas mais realistas.

O instrumento descreve orientações e apresenta dois formulários: o primeiro, para as informações gerais sobre o estabelecimento de saúde; e o segundo, subdividido em localização geográfica, aspectos estruturais (colunas, vigas, muros, lajes, fundações e outros), aspectos não estruturais (redes elétricas, hidráulicas, sanitárias, os sistemas de ventilação, ar condicionado, entre outros; o mobiliário e os equipamentos de escritórios fixos ou móveis, assim como os equipamentos médicos e de laboratório, suprimentos utilizados para o diagnóstico e tratamento, além dos elementos arquitetônicos da edificação, entre outros) e aspectos de funcionalidade (organização técnica e administrativa de seu pessoal para responder às situações: plano de emergência, plano de contingência, educação permanente, entre outros) $)^{3,14}$.

O ISH global varia de 0 a 1 . De acordo com a pontuação, o hospital é classificado em uma das três categorias de risco: $\mathrm{C}(0$ a 0,35$)-$ existe a possibilidade de o hospital deixar de 
funcionar em caso de desastre, com prejuízo para a vida de profissionais e de usuários; $\mathrm{B}$ (de 0,36 a 0,65) - o hospital mantém-se em atividade com riscos para materiais e equipamentos, podendo comprometer a ação de profissionais e pôr em risco a capacidade de atendimento e o potencial do hospital; e A (de 0,66 a 1) - mesmo em situações de desastres, provavelmente o hospital continuará funcionando, pois protege a vida de profissionais e usuários. O ISH é calculado automaticamente, por meio das respostas lançadas em uma planilha de Excel ${ }^{\circledR 3}$.

Os valores obtidos pelo ISH não se constituem valores definitivos da capacidade do estabelecimento de saúde, pois isso requer estudos detalhados que incluem: estudo de ameaças, de vulnerabilidade estrutural, de vulnerabilidade não estrutural e de vulnerabilidade organizativo-funcional, porém estes, apesar de grande importância, são complexos e onerosos, de forma que nem sempre são viáveis ${ }^{17,18}$.

Países de língua espanhola das Américas do Sul, Central e do Norte vêm adotando o ISH concebido pela Opas em razão de fenômenos naturais terem provocado desastres no passado e do risco de poderem produzi-los no futuro. No México, muitos hospitais foram reformados, e outros tantos são construídos tendo esse instrumento de avaliação como referência ${ }^{18}$.

As unidades de saúde, especialmente os hospitais, diante da vulnerabilidade no contexto dos desastres, assumem relevância por agregarem valor econômico ao seu irrefutável valor social. É imprescindível que permaneçam seguras para poderem fazer frente às demandas das pessoas atingidas por desastres ${ }^{4}$.

O ISH destaca-se como ferramenta de planejamento; e, alinhado com as ações de gestão de risco preconizadas pelo Marco de Sendai, fornece informações de avaliação para se estabelecerem: ações a desenvolver, prazos, prioridades, responsabilidades dentro da instituição, e identifica os recursos necessários para sua implementação (plano de intervenção). Hoje, ele é um dos instrumentos de maior aplicação no mundo e estádisponível em inglês, espanhol, árabe, russo, francês e português ${ }^{\mathbf{1 4 , 1 7}}$.
Desde 2008, o ISH tem sido utilizado por países da América Latina (Bolívia, Equador, Chile e Peru), Caribe (Montserrat, São Vicente e Granadinas, Anguilla, Dominica, Barbados), México e outros?.

Em Cuba, a aplicação do ISH resultou na capacidade funcional operante dos hospitais, mesmo após a passagem de furacões devastadores, tais como o ‘Gustav' e o 'Ike’ em 2008. Em março de 2012, 31 países e territórios das Américas relataram seu uso para estabelecer prioridades, sendo um dos elementos centrais na implementação de políticas e programas nacionais e subnacionais para hospitais seguros. Mais de 1.400 hospitais nas Américas foram avaliados com o ISH, e determinou-se que: $51 \%$ estavam na categoria $\mathrm{A}$, ou seja, com uma alta probabilidade de continuar a funcionar em caso de desastre; $37 \%$ estavam na categoria $\mathrm{B}$, sendo provável que não colapsassem, mas provavelmente deixariam de funcionar; e $12 \%$ se encontravam na categoria $\mathrm{C}$, indicando que provavelmente entrariam em colapso, deixando de funcionar e não garantindo a vida dos pacientes e da equipe na vigência do desastre19.

Representantes da Organização Mundial da Saúde, com integrantes de diversas nações, encontraram-se na Turquia, ainda no ano de 2012, para revisar os instrumentos de avaliação de segurança hospitalar utilizados nos diferentes continentes e concordaram em usar o ISH como base para gerar um instrumento de aplicação global, que possa ser adaptado às distintas realidades. Algumas regiões do mundo o adotaram originalmente, e outras o adaptaram ao seu próprio contexto ${ }^{\mathbf{1 4}, 19}$.

No relatório final do plano estratégico da Opas referente ao período 2008-2013, afirma-se que o ISH foi aplicado em mais de 2.900 hospitais e outros serviços de saúde em 33 países e territórios, possibilitando a implantação de medidas corretivas necessárias em diversos estabelecimentos de saúde ${ }^{20}$.

Para aplicar o ISH, recomenda-se a composição de uma equipe multiprofissional, sob a abrangência de distintos saberes, incluindo a engenharia, a arquitetura, a medicina, 
a enfermagem, a administração e suas diversas modalidades. A articulação dos saberes, que interagem entre si, amplia a visão de cada um deles acerca de um determinado aspecto da vivência profissional, pois agrega dimensões e enfoques pouco explorados. Os profissionais devem estar relacionados com o setor da construção de estabelecimentos de saúde, da assistência de saúde, de administração ou de atividades de suporte, como manutenção e outras. Preferencialmente, os avaliadores devem ter um mínimo de cinco anos de experiência em projeto estrutural, construção ou gerenciamento hospitalar em desastres ${ }^{3,14}$.

O ISH é um primeiro e importante passo para os países, a fim de privilegiar investimentos para a melhoria de segurança das suas instalações de saúde. Em termos comparativos, é como tirar uma fotografia do hospital, que fornece os elementos básicos necessários para identificar as características do estabelecimento e confirmar ou descartar a presença de riscos iminentes, como um perfil instantâneo de uma unidade de saúde que poderá dar continuidade às suas funções durante situações de emergência, baseado em fatores estruturais, não estruturais e funcionais, incluindo o ambiente, as pessoas e as redes de serviços de saúde as quais pertencem²1.

Devido à importância constatada no uso do ISH para a gestão de riscos no setor saúde, o instrumento foi aperfeiçoado para sua aplicação em hospitais de pequeno porte a partir de 2010, sendo na nova versão indicado para estabelecimentos de até 20 leitos ${ }^{17}$.

A partir do ano de 2012, buscando proporcionar melhorias nas condições dos estabelecimentos de saúde e com o objetivo de aperfeiçoar o conceito de hospital seguro, a Opas iniciou o projeto 'hospitais inteligentes', visando conciliar os seguintes conceitos: redução de custos com serviços de saúde e serviços públicos, redução das emissões de Gases de Efeito Estufa (GEE), melhor qualidade do ar, maior acesso físico aos hospitais, melhor acesso à água segura e melhores condições de segurança ${ }^{22}$.
No final do ano de 2015, a Opas consolidou o projeto 'hospitais inteligentes', apresentando resultados satisfatórios de sua utilização nos países caribenhos, e lançou o 'kit de ferramentas para hospitais inteligentes', o 'Smart Hospitals Toolkit'. Este guia, composto pelo ISH e por orientações e instruções sobre normas técnicas para hospitais inteligentes, busca possibilitar que a unidade de saúde alcance seus melhores resultados, conciliando custo-benefício, sustentabilidade e segurança ${ }^{22}$.

No Brasil, o tema 'hospitais seguros frente a desastres' e a aplicação do ISH vêm sendo tratados ainda de maneira incipiente, com tímidas iniciativas em produções científicas e ainda não incorporados plenamente ao contexto brasileiro. O movimento para tradução e aproximação cultural do ISH foi desenvolvido ${ }^{\mathbf{1 4}}$, mas ainda se faz necessária a sua validação como instrumento para efetiva utilização por equipes multiprofissionais.

Um estudo publicado no Brasil, versando sobre a aplicação do ISH, revelou o preocupante cenário em relação à política de hospitais seguros no País, contrariando o preconizado pela Opas e evidenciando que é possível a aplicação do ISH nos hospitais brasileiros. Nele, os autores buscaram estimar o ISH para três hospitais da região noroeste do estado do Rio de Janeiro, área regularmente atingida por desastres de origem natural. Os três hospitais avaliados apresentaram classificação de risco intermediário (nível B do ISH). Os componentes estruturais foram os que apresentaram melhores resultados nos três hospitais. Os autores concluíram que existem desafios políticos e institucionais para a efetivação da política de hospitais seguros no Brasil9.

Em contraposição à importância estratégica do ISH no intuito de colaborar para o desenvolvimento de ações que preparem o estabelecimento de saúde para os impactos de desastres, percebe-se que, no Brasil, tanto o instrumento quanto a temática envolvendo hospitais seguros mostram-se 
praticamente inexpressivos, o que é resultante da cultura brasileira de não se antecipar aos acontecimentos.

Entender o panorama relacionado com as ocorrências dos eventos adversos é fundamental para o planejamento das ações de prevenção e controle. A gestão de riscos de desastres ocorre por meio de um conjunto de medidas mitigadoras, que contemplam desde o planejamento até a execução de atividades pontuais e coletivas realizadas por equipes coordenadas e integradas por representantes de diversas áreas de conhecimento.

Por isso, a interdisciplinaridade e a interprofissionalidade revelam-se prioritárias para a efetivação das tecnologias empregadas no processo de gestão dos desastres. Conjugar esforços e propiciar o entrosamento de vivências e conhecimentos diversificados nesse cenário desafiador para o homem possibilitam a antecipação necessária, para que sejam evitadas inúmeras perdas e amenizadas muitas dores.

Há, por certo, muitos desafios a serem enfrentados para que seja realmente alcançado um modelo de trabalho que conjugue e articule diversas áreas de saber, atuando no contexto dos desastres. Ademais, ainda é utópico pensarmos em transdisciplinaridade no caso de atendimento em desastres. Todavia, buscar o 'trans' é importante para que se alcance um modelo ideal de uma condição de atuação colaborativa e sem fronteiras entre várias áreas de conhecimento. Os desafios para a interdisciplinaridade são os mesmos que vamos descobrir na transdisciplinaridade, porém, ainda de forma mais exacerbada, já que essa proposta visa, efetivamente, à comunicação real entre os diferentes campos de saber. A transdisciplinaridade vai além da interdisciplinaride, pressupondo uma postura completamente aberta, sem restrições, sem julgamentos; nesse sentido, é um trabalho que exige que os comprometidos com a proposta possam manter uma relação de aceitação e compreensão do próximo, criando as condições e tecnologias necessárias ao bom trabalho em equipe ${ }^{\mathbf{1 2}}$.

\section{Considerações finais}

A política de hospitais seguros enunciada pela Opas representa um importante marco no contexto da gestão de risco de desastres. $\mathrm{O}$ sucesso da campanha traduz-se em sua ampla aplicação no mundo, e mais fortemente na América Latina.

A interdisciplinaridade e a interprofissionalidade para a consecução de hospitais seguros mostram-se fundamentais, fazendo com que diversos agentes atuem em conjunto, para que, assim, por meio de trocas de experiências e compartilhamento de saberes, os melhores resultados sejam alcançados.

Compreender a multidimensionalidade dos desastres possibilita o diagnóstico urgente de que, para lidar com eles, é indispensável o debate interdisciplinar e interprofissional. É urgente o engajamento dos diversos atores sociais.

Todo esforço precisa ser dedicado no planejamento para lidar com um tema tão caro para a humanidade. Os desastres não podem ser ignorados. Sua ocorrência está fortemente associada à condição de vida atual das populações, muitas vezes negligenciadas pelo poder público e em desalinho com a preservação do meio ambiente.

Medidas precisam ser implementadas, reforçadas e multiplicadas para que a sociedade esteja em melhores condições para evitar e, se for o caso, atuar com eficiência antes, durante e após os desastres.

O setor saúde, dada a sua condição estratégica no contexto social e, particularmente, na ocorrência de emergências e desastres, necessita desenvolver estratégias e ações que lhe permitam o pleno funcionamento no momento em que a população mais precisa buscar o socorro nas unidades de saúde.

Em consonância com a necessidade de manter preparados os estabelecimentos de saúde para que cumpram sua finalidade, inclusive em condições adversas, é indispensável avançar em pesquisas sobre o tema 'hospitais seguros frente a desastres', o que se coaduna com os apontamentos do Marco 
de Sendai (2015-2030) para a Redução de Risco de Desastres.

Nessa perspectiva, o ISH destaca-se como importante instrumento de aplicação interdisciplinar para avaliar a concepção de hospitais seguros, uma vez que quantifica e estratifica os dados obtidos, constituindo-se como uma ferramenta norteadora para a tomada de decisões dos gestores no tocante à elegibilidade das ações de investimento em segurança hospitalar, reduzindo ou eliminando as vulnerabilidades estruturais e não estruturais do hospital, aumentando sua capacidade de resistência aos desastres. Apesar de não substituir o estudo de vulnerabilidade, o ISH oferece uma orientação inicial e referência para que possam ser estabelecidas prioridades de investimentos na otimização da segurança nas instalações de saúde.

Por fim, podemos assegurar que, para promover os melhores estudos, ações e estratégias voltadas para a redução de riscos de desastres,é prioritário que se incentive a consolidação da política de hospitais seguros, e que isso seja fundamentado na mais ampla discussão interdisciplinar, em práticas interprofissionais colaborativas e na efetiva participação da sociedade, com vistas à consolidação da cultura de segurança em estabelecimentos de saúde no Brasil.

\section{Colaboradores}

Cardoso RBS (0000-0002-0810-4571)* desenvolveu a concepção e elaboração do manuscrito. Oliveira AB (0000-0003-4611-1200)* desenvolveu a concepção, revisão crítica do conteúdo e aprovação da versão final do manuscrito.

\section{Referências}

1. Freitas CM, Silva DRX, Sena ARM, et al. Desastres naturais e saúde: uma análise da situação do Brasil. Ciênc. Saúde Colet. [internet]. 2014 [acesso em 2019 jun 1]; 19(9):3645-56. Disponível em: http://www.scielo.br/pdf/csc/v19n9/1413-8123-csc-19-09-3645.pdf.

2. United Nations Office for Disaster Risk Reduction. Sendai Framework for Disaster Risk Reduction 20152030 [internet]. Genebra: United Nations; 2015. [acesso em 2019 jun 1]. Disponível em: https://www.unisdr.org/we/coordinate/sendai-framework.
3. World Health Organization, Pan American Health Organization. Hospital Safety Index [internet]. Washington, DC: WHO/OPS; 2008. [acesso em 2019 mar 1]. Disponível em: http://iris.paho.org/xmlui/ bitstream/handle/123456789/812/SafeHosEvaluatorGuideEng.pdf?sequence=1\&isAllowed $=y$.

4. Organización Mundial de la Salud, Organización Panamericana de la Salud. Hospitales seguros ante inundaciones [internet]. Washington, DC: WHO; OPS; 2006 [acesso em 2019 mar 1]. Disponível em: https://
${ }^{\star}$ Orcid (Open Researcher and Contributor ID). 
www.paho.org/disasters/index.php?option=com d o c m an \& vi ew = d own lo ad \& c a t e gory slug=books\&alias=1803-hospitales-seguros-ante-inundaciones-capitulo-1\&Itemid=1179\&lang=en .

5. Organización Panamericana de la Salud. ¿̇u hospital es seguro? Preguntas y respuestas para el personal de salud [internet]. Quito: OPS; 2007. [acesso em 2020 jun 20]. Disponível em: http://cidbimena.desastres.hn/docum/crid/HospitalesSeguros/pdf/spa/ doc16726/doc16726.htm.

6. Cerrutti DF, Oliveira MLC. Aplicação da gestão de risco de desastres no Sistema Único de Saúde (SUS). Cad. Saúde Colet. [internet]. 2011 [acesso em 2019 jun 1]; 19(4):417-24. Disponível em: http://bvsms.saude. gov.br/bvs/publicacoes/aplicacao_gestao_risco_desastres_sus.pdf.

7. Wisner B, Gaillard JC, Kelman I. Framing disaster: theories and stories seeking to understand hazards, vulnerability and risk [internet]. In: Wisner B, Gaillard JC, Kelman I, editores. Handbook of hazards and disaster risk reduction. Abingdon; Oxford shire: Routledge Hand Book; 2012. p. 18-33. [acesso em 2019 mar 1]. Disponível em: https://www.researchgate. net/publication/311535065_Framing_disaster_theories_and_stories_seeking_to_understand_Hazards_vulnerability_and_risk.

8. Saba LCP, Cardoso TAO, Navarro MBMA. Hospital seguro frente aos desastres: uma reflexão sobre biossegurança e arquitetura. Rev Panam Salud Publica [internet]. 2012 [acesso em 2019 jun 1]; 31(2):176-80. Disponível em: https://www.scielosp.org/article/ rpsp/2012.v3ln2/176-180/.

9. Salles MJP, Cavalini LT. Implementação da 'estratégia de hospitais seguros frente a desastres' no Brasil: desafios e evidências empíricas. Hygeia [internet]. 2012 [acesso em 2019 jun 1]; 14(8):81-90. Disponível em: http://www.seer.ufu.br/index.php/hygeia/article/view/17109.

10. Londe LR, Marchezini V, Conceição RS, et al. Impactos de desastres socioambientais em saúde pública: estudos dos casos dos Estados de Santa Catarina em 2008 e Pernambuco em 2010. Rev. bras. estud. Popul. [internet]. 2015 [acesso em 2019 jun 13]; 32(3):537-62. Disponível em: http://www.scielo.br/scielo.php?script=sci arttext\&pid=S0102-30982015000300537.

11. Bankoff G, Frerks G, Hilhorst D, editores. Mapping vulnerability: disasters, development and people. London; New York: Routledge Handbook; 2004.

12. Carmo R, Valêncio N, organizadores. Segurança humana no contexto dos desastres [internet]. São Carlos: RiMa; 2014. [acesso em 2019 jun 13]. Disponível em: http://www.nepo.unicamp.br/publicacoes/livros/segurancahumana/segurancahumana.pdf.

13. Valencio N, Valencio A. O assédio em nome do bem: dos sofrimentos conectados à dor moral coletiva de vítimas de desastres. Lumina [internet]. 2018 [acesso em 2019 jun 1]; 12(2):19-39. Disponível em: https://periodicos.ufjf.br/index.php/lumina/article/ view/21531.

14. Cardoso RBS. Hospitais seguros frente a desastres: tradução e aproximação cultural do Indice de Seguridad Hospitalaria para o contexto brasileiro [dissertação] [internet]. Rio de Janeiro: Escola de Enfermagem Anna Nery, Universidade Federal do Rio de Janeiro; 2018. 235 p. [acesso em 2019 jun 1]. Disponível em: http://objdig.ufrj.br/51/teses/870111.pdf.

15. Folly M. Migrantes invisíveis: a crise de deslocamento forçado no Brasil: 2018. Instit. Igarapé [internet]. 2018 [acesso em 2019 jun 1]; (29):1-33. Disponível em: https://igarape.org.br/wp-content/uploads/2018/03/ Migrantes-invis\%C3\%ADveis.pdf.

16. Fujita DM, Salvador FS, Damião GPS, et al. Increase of immigrants in emerging countries: free public healthcare and vaccination as preventive measures in Brazil. Cad. Saúde Pública [internet]. 2019 [acesso em 2019 jun 1]; 35(2):e00228118. Disponível em: https:// www.scielosp.org/pdf/csp/2019.v35n2/e00228118/ en

17. Organización Mundial de la Salud, Organización Panamericana de la Salud. Indice de seguridad hospitalaria: guía para evaluadore. 2. ed. [internet]. Washington, 
DC: OMS; OPS; 2018. [acesso em 2020 jun 20]. Disponível em: https://iris.paho.org/handle/10665.2/51462.

18. Pereira CAR, Barata MML. Organização dos serviços urbanos de saúde frente à mudança do clima e ao risco de desastres na América Latina. Saúde debate [internet]. 2014 [acesso em 2019 jun 1]; 38(102):624-34. Disponível em: http:// www.scielo.br/scielo.php?script=sci_arttext\&pid =S0103-11042014000300624

19. World Health Organization, Pan American Health Organization. Emergencies News. The Safe Hospitals Initiative in the world [internet]. Washington, DC: WHO; OPS; 2018. [acesso em 2019 mar 1]. Disponível em: http://www.paho.org/disasters/newsletter/index. php?option $=$ com $\_$content $\&$ view $=$ article $\&$ id $=495$ :la -iniciativa-de-hospitales-seguros-en-el-mundo\&cat id=232\&Itemid=303\&lang=es.

20. Cooperazone Internazionale. Hospitais seguros: práticas fundamentais para implementadores de RRC [in- ternet]. Roma: COOPI; 2014. [acesso em 2019 mar 1]. Disponível em: http://www.fao.org/3/a-i3770o.pdf.

21. Colombia. Ministerio de Salud y Protección Social. Programa hospitales seguros frente a desastres [internet]. Bogotá, DC: Ministerio de Salud y Protección Social; 2017. [acesso em 2019 mar 1]. Disponível em: https://minsalud.gov.co/salud/PServicios/Paginas/programa-hospitales-seguros.aspx.

22. World Health Organization, Pan American Health Organization. Smart Hospitals Toolkit [internet]. Washington, DC: WHO; OPS; 2017. [acesso em 2019 mar 1]. Disponível em: https://www.paho.org/disasters/ index.php?option=com_content $\&$ view $=$ article $\&$ id $=1$ 742:smart-hospitals-toolkit\&Itemid=1248\&lang=en.

Recebido em 30/09/2019

Aprovado em 29/02/2020

Conflito de interesses: inexistente

Suporte financeiro: não houve 